\title{
Community acquired pneumonia: aetiology and prognostic index evaluation
}

\author{
N C Karalus, R T Cursons, R A Leng, C B Mahood, R P G Rothwell, B Hancock, \\ $S$ Cepulis, $M$ Wawatai, L Coleman
}

\begin{abstract}
A 10 month prospective study of all adults admitted to Waikato Hospital with community acquired pneumonia was performed to assess aetiology, mortality, hospital stay, and the value of a prognostic index based on that obtained from a British Thoracic Society study. The 92 patients in the survey had a mean age of 56 (range 13-97) years. A microbiological diagnosis was established in 72\%: Streptococcus pneumoniae (33\%), Mycoplasma pneumoniae (18\%), and influenza $A$ virus $(8 \%)$ were the most common microorganisms. Other causative organisms were Legionella pneumophila (4 cases), Staphylococcus aureus (3), Klebsiella pneumoniae (2), Haemophilus influenzae (2), Nocardia brasiliensis (1), and Acinetobacter calcoaceticus (1). Chlamydia sp, influenza $B$ virus and adenovirus were each found in one case; all were cultured on nasopharyngeal aspirates. Aspiration was considered to be the underlying cause in five patients, two with epilepsy and one with pseudobulbar palsy. Five of the six deaths that occurred were in patients over 75 years of age and the other was 69. In four of the six the established causative organisms were Chlamydia sp (1), K pneumoniae (1), and $S$ aureus (2). Patients had a 16 fold increased risk of death if they had two or more of the following on admission: a respiratory rate of $30 /$ minute or more, diastolic blood pressure of $60 \mathrm{~mm} \mathrm{Hg}$ or less, and either confusion or a plasma urea concentration greater than $\mathbf{7 \cdot 0}$ $\mathbf{m m o l} / \mathbf{l}$.
\end{abstract}

On the basis of the findings of a prospective study of community acquired pneumonia in 25 British hospitals, the British Thoracic Society recommended that antibiotics should be given as early as possible and chosen always to cover Streptococcus pneumoniae and in addition Mycoplasma pneumoniae during outbreaks and Staphylococcus aureus during influenza epidemics. ${ }^{1}$ Adults aged 74 years or more were not included in the study, though the elderly comprise a sizable proportion of adults admitted to our hospital with pneumonia. The study also developed a prognostic index that identified patients whose risk of death was 21 times greater than that of patients not satisfying the prognostic criteria. A prospective study of all adults with com- munity acquired pneumonia admitted to Waikato Hospital was therefore undertaken. We also prospectively assessed an index applied within 24 hours of admission in a group of adult patients, which included those over 74 years old. We based our investigation on the British Thoracic Society study, using the same microbiological criteria, though we also assessed the impact of Chlamydia species in the aetiology of pneumonia. Our intention was to develop a protocol of investigation and management, a key feature of which would be identification of high risk patients, early identification of causative organisms, and prognostic evaluation to guide treatment.

\section{Methods}

Pneumonia was defined as an acute respiratory illness with radiographic pulmonary shadowing that was at least segmental or present in more than one lobe and was neither pre-existing nor of other known cause. All patients admitted to a medical ward from February to November 1988 were eligible. Patients were excluded prospectively when pneumonia was not the main reason for hospital admission or was an expected terminal event, when the pneumonia was distal to bronchial obstruction due to a foreign body or neoplasm, or when it was due to Mycobacterium tuberculosis. Patients with bronchiectasis were included if the chest radiograph showed new consolidation.

Clinical data on the history of the illness, personal details, past medical history, alcohol intake, and cigarette consumption were recorded on standard forms. Clinical findings at admission were recorded by the admitting medical registrar.

The chest radiographs were later assessed by investigators CBM, RPGR, and LTC for the presence of consolidation in one lobe only or two or more lobes, of pleural effusion, and of cavitation. Antibiotic use was determined by the admitting physician. The number of days spent in hospital (three very elderly patients were subsequently transferred to a rehabilitation unit before hospital discharge), outcome, and clinically relevant complications were recorded. Arterial blood gas tensions (during the breathing of air); plasma concentrations of sodium, potassium, urea, and albumin; and a full blood count were obtained at admission.

The following microbiological tests were performed: two blood cultures, sputum examination (Gram staining, culture and sensitivity), pneumococcal antigen detection by latex agglutination $(\mathrm{pH} 7 \cdot 2$; Wellcome Diag- 
Table 1 Criteria for microbiological diagnosis

\begin{tabular}{|c|c|c|}
\hline Organisms & Definite & Probable \\
\hline Streptococcus pneumoniae & $\begin{array}{l}\text { Blood culture } \\
\text { Urinary antigen }\end{array}$ & Sputum culture or antigen \\
\hline Other bacteria & Blood culture & Sputum culture \\
\hline Legionella species & $\begin{array}{l}\text { Nasopharyngeal aspirate or } \\
\text { sputum culture or direct } \\
\text { fluorescent antibody } \\
\text { Fourfold antibody rise }\end{array}$ & \\
\hline Mycoplasma pneumoniae & Fourfold antibody rise & $\begin{array}{l}\text { Single agglutination titre } \\
\geqslant 640 \\
\text { Single complement fixation } \\
\text { test titre } \geqslant 128\end{array}$ \\
\hline Chlamydia species & $\begin{array}{l}\text { Nasopharyngeal aspirate } \\
\text { culture } \\
\text { Fourfold antibody rise }\end{array}$ & \\
\hline Viruses & $\begin{array}{l}\text { Nasopharyngeal aspirate } \\
\text { culture } \\
\text { Fourfold antibody rise }\end{array}$ & \\
\hline
\end{tabular}

nostics) and countercurrent immunoelectrophoresis ( $\mathrm{pH} 8.6$; Omniserum, Statens Seruminstitut, Copenhagen), and direct fluorescent antibody tests for Legionella pneumophila (Genetic Systems). Sputum samples with less than 10 polymorphonuclear leucocytes per squamous cell were not processed. Sputum was digested with an equal volume of $2 \% \quad N$-acetyl-L-cysteine for 15 minutes, boiled for five minutes, and centrifuged at $10000 \mathrm{~g}$ for five minutes. The supernatant was used for pneumococcal antigen determination. Nasopharyngeal aspirates (obtained by $M W$ ) were submitted for culture of viruses, Chlamydia, and Legionella and for direct fluorescent antibody tests. ${ }^{18}$ Urine obtained at admission was concentrated by ethanol precipitation and frozen for pneumococcal antigen determination. Acute and convalescent serum was obtained for determination of titres of antibody to Legionella species (pneumophila serogroups 1-6, bozemanii 1 and 2, dumoffi, gormanii, micdadei, longbeachae 1 and 2, and jordanis). Indirect

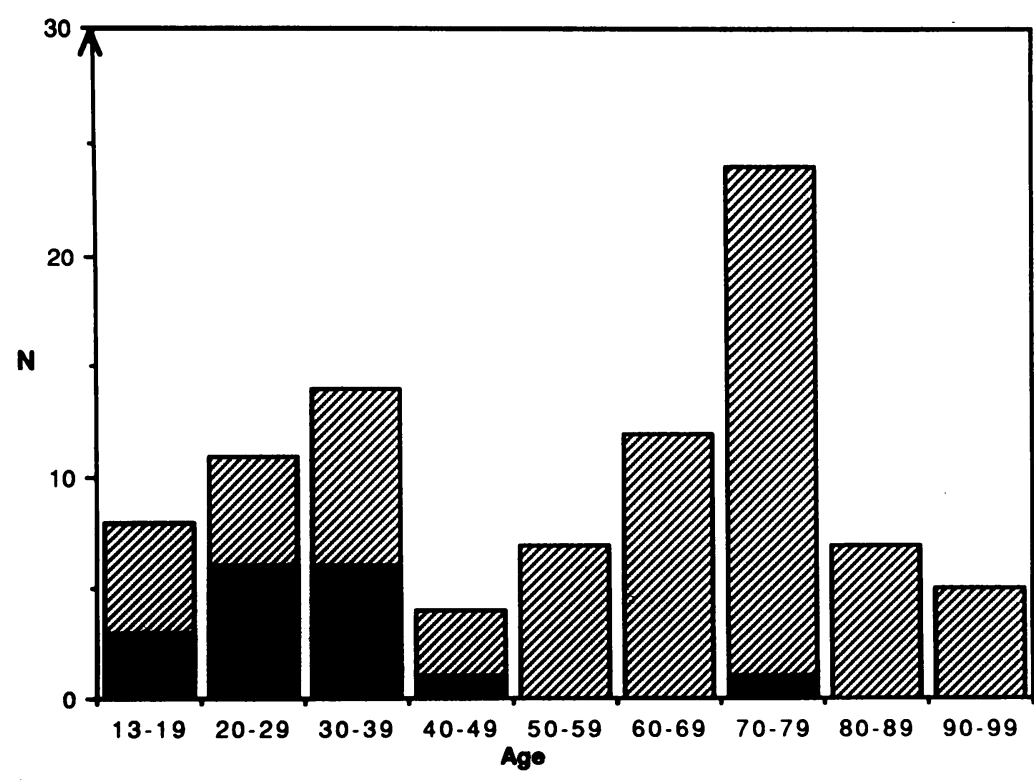

Age distribution of the patients. $\mathbb{Z}$ All causative organisms; $\square$ Mcoplasma pneumoniae. fluorescent antibody positive results were confirmed by the National Health Institute, Wellington, influenza $A$ and $B$ virus and adenovirus (CFT-Hoechst), Chlamydia species (Ipazyme) and Mycoplasma pneumoniae (CFT-Hoechst, gel-particle agglutinationSerodia). Coxiella burnetti does not occur naturally in New Zealand and was not sought.

Chlamydia species were sought by culture of nasopharyngeal aspirates in both Hela 229 and BGM cell lines and identified by using genus specific FITC labelled monoclonal antibodies (Bartel Diagnostics). Serological tests were developed with the new species Chlamydia pneumoniae (strain TWAR), obtained from the Washington Research Foundation, and Chlamydia trachomatis species LGV II as antigens. These were used to infect BGM cells to produce inclusion body antigen slides. Patients' serum was then screened by the indirect fluorescent antibody technique. Positive serological results were confirmed by Professor J T Grayston, University of Washington, on the basis of specific microimmunofluorescence.

The criteria for microbiological diagnosis are given in table 1 .

In the British Thoracic Society study a prognostic index was developed by multiple regression analysis. A 21 fold increase in risk of death was present in patients with two or more of the following: supine diastolic blood pressure at admission $60 \mathrm{~mm} \mathrm{Hg}$ or less, respiratory rate at admission $30 /$ minute or more, plasma urea concentration greater than $7 \cdot 0 \mathrm{mmol} / \mathrm{l}$ during the hospital stay. Confusion at admission could be substituted for raised plasma urea concentration, allowing immediate application of this index to patients at admission. Because we wanted an index that may be helpful in determining treatment we used plasma urea concentration determined on admission or in the first 24 hours, so that serum urea or confusion could be used as the third factor in the index.

\section{Results}

\section{CLINICAL FINDINGS}

Ninety two patients were studied during the 10 months of the survey. Ages ranged from 13 to 97 (mean 56) years, with 32 patients aged over 74 (fig). Those with $M$ pneumoniae were younger. Men predominated (53:39). There were six deaths $(6.5 \%)$, all but one (aged 69 ) in those over 74 . Eight were living in rest homes and the remainder in private dwellings. The mean duration of hospital stay was 8.65 days, and 42 $(45 \%)$ were admitted in the winter during June, July, and August.

Chronic illness was present in $54(59 \%)$ and was of many varieties (19 had chronic airflow obstruction); 37 of these patients were over 64 years and 24 were over 74 . Four had definite immune suppression. Thirty five had a smoking history of 20 or more pack years and 11 were drinking alcohol daily.

Of the 92 patients, 47 had received antibiotics before admission, including 15 of the 17 infected with $M$ pneumoniae. Nearly a 
Table 2 Radiographic findings in community acquired pneumonia by microbiological cause

\begin{tabular}{lllll}
\hline & One lobe & $\begin{array}{l}\text { Two or } \\
\text { more lobes }\end{array}$ & Pleural fluid & Cavity \\
\hline Mycoplasma pneumoniae & 13 & 4 & 1 & 0 \\
Streptococcus pneumoniae & 23 & 7 & 3 & 0 \\
Other organisms & 12 & 6 & 2 & 1 \\
Unknown aetiology & 19 & 8 & 4 & 1 \\
Total & 67 & 25 & 10 & 2 \\
\hline
\end{tabular}

third of patients denied sputum production. Sputum was obtained from $75(82 \%)$ of patients (every effort was made to obtain sputum in the first three hours after admission).

Pleuritic chest pain was common $(44,48 \%)$, but a pleural rub was infrequently documented $(10,11 \%)$; there was an impression that pleuritic pain was less frequent with $M$ pneumoniae aetiology $(22,23 \cdot 5 \%)$.

Confusion was evident at the time of admission in 24 patients $(26 \%)$. Sixteen of these 24 were over 75 years of age and only two less than 65.

Radiographic findings are summarised in table 2. Cavitation occurred in two cases-the patient with Nocardia brasiliensis and a young epileptic woman who had no microbiological diagnosis but had had a grand mal seizure four days before admission.

The modified British Thoracic Society prognostic index predicted five of the six deaths. Altogether 22 patients were at high risk; five died (positive predictive value $22 \cdot 7 \%$ ). One of the remaining 70 died $(1.4 \%)$, so that the prognostic index indicated a relative risk of death of 16.2 in this older population. The prognostic index for prediction of outcome was significant $\left(p<0.01, \chi^{2}\right.$ test with Yates's correction). Of the 30 over 74 years of age, 12 had a bad prognostic index and five of the 12 died; no one with a good prognosis died. Of those under 75, 10 had a bad prognostic index but the only death occurred in a patient with a good index. No patient with $M$ pneumoniae had a bad prognostic index.

Arterial blood gases were obtained at admission from 84 patients, 14 of whom were having

Table 3 Microbiological diagnoses

\begin{tabular}{llcc}
\hline & Aetiology & & \\
\cline { 2 - 4 } & Definite & Probable & Total \\
\hline Streptococcus pneumoniae & 11 & 19 & 30 \\
Mycoplasma pneumoniae & 17 & & 17 \\
Influenza A virus & 8 & & 8 \\
Legionella species & 4 & & 4 \\
Chlamydia species & 2 & 1 & 2 \\
Staphylococcus aureus & 2 & 1 & 2 \\
Klebsiella pneumoniae & 1 & & 1 \\
Acinetobacter species & 1 & & 1 \\
Nocardia brasiliensis & 1 & & 1 \\
Influenza B virus & 1 & 5 & 5 \\
Adenovirus & 1 & 1 & 1 \\
Haemophilus influenzae & 0 & 1 & 0 \\
Escherichia coli & 0 & 0 & 77 \\
Serratia marcescens & 0 & 28 & $(20) \dagger$ \\
Respiratory syncytial virus & 0 & & \\
& $49 \quad(47)^{\star}$ & & \\
\hline
\end{tabular}

$\star$ Number of patients.

†Number of additional patients. supplementary oxygen. Despite this $43(51 \%)$ had hypoxaemia $\left(\mathrm{PaO}_{2} \leqslant 60 \mathrm{~mm} \mathrm{Hg}(8.0 \mathrm{kPa})\right)$, including seven who were having supplementary oxygen. No patient required assisted ventilation. Of the 77 who had plasma urea concentration estimated at admission, 39 $(51 \%)$ had a value greater than $7.0 \mathrm{mmol} / \mathrm{l}$.

Leucocytosis at admission (white blood cells $\left.\geqslant 11.0 \times 10^{9} / 1\right)$ was common $(63,68 \%)$, being almost invariable in those with pneumococcal infection $(86,93 \%)$ but less frequent among those with $M$ pneumoniae infection $(38,41 \%)$.

\section{MICROBIOLOGY}

The microbiological diagnoses are summarised in table 3. In $47(51 \%)$ patients a definite microbiological diagnosis was established and a further 28 probable diagnoses were made (which added 20 patients). Overall a microbiological diagnosis was made in 67 patients $(72 \%), 10$ of whom had two infective organisms.

Blood cultures were obtained at admission in 87 patients, and in eight this was positive for $S$ pneumoniae (including one patient having oral erythromycin). A positive culture was obtained for $S$ aureus, Acinetobacter calcoaceticus, and Klebsiella pneumoniae from one patient each.

Sputum was obtained from 75 patients at admission, culture of which indicated a probable pathogen in 23 patients: $S$ pneumoniae 13, $H$ influenzae $5, S$ aureus $2, K$ pneumoniae 2 ; and Acinetobacter sp, Escherichia coli, and Serratia marcescens one each. Four of these patients had an identical organism on the blood culture and this was considered definitely aetiological ( $S$ pneumoniae, $S$ aureus, $A$ calcoaceticus, $K$ pneumoniae). A further patient had sputum cultures repeatedly positive for $N$ brasiliensis, and this finding was considered aetiologically "definite". Three of the above patients had both $S$ pneumoniae and H influenzae cultured in sputum, and so these are classified as having dual infections.

Sixty one sputum samples were studied for pneumococcal antigens, all by latex agglutination and most by countercurrent immunoelectrophoresis. Twenty two were positive by latex agglutination; three of these were not confirmed by countercurrent immunoelectrophoresis and four of the 22 did not have immunoelectrophoresis performed because samples were inadvertently discarded. Nineteen were positive according to immunoelectrophoresis, four of these not being confirmed by latex agglutination. If we take a positive result in either test as a probable infection, 26 patients had sputum containing pneumococcal antigen. The detection of pneumococcus is summarised in table 4. No patient with $M$ pneumoniae infection had pneumococcal antigen detected in sputum or urine. Sputum antigen tests provided the only indication of pneumococcal infection in six patients.

Nasopharyngeal aspirates were obtained from 83 patients $(90 \%)$. Five positive cultures were obtained: two for influenza $A$ and one each for influenza B, adenovirus, and Chlamydia sp. The Chlamydia sp could not be passaged, so the strain could not be conclusively identified. The 
Table 4 Detection of pneumococci in 30 patients

\begin{tabular}{|c|c|c|c|c|c|c|c|}
\hline \multirow[b]{3}{*}{ Method } & \multicolumn{7}{|c|}{ Numbers of patients } \\
\hline & \multirow[b]{2}{*}{ Positive } & \multirow{2}{*}{$\begin{array}{l}\text { With other } \\
\text { positive } \\
\text { specimens }\end{array}$} & \multicolumn{5}{|c|}{ Positive by other methods } \\
\hline & & & $\begin{array}{l}\text { Blood } \\
\text { culture }\end{array}$ & $\begin{array}{l}\text { Urine } \\
\text { CIE }\end{array}$ & $\begin{array}{l}\text { Sputum } \\
\text { Gram stain }\end{array}$ & $\begin{array}{l}\text { Sputum } \\
\text { culture }\end{array}$ & $\begin{array}{l}\text { Sputum } \\
\text { antigen }\end{array}$ \\
\hline Blood culture & 8 & 3 & (8) & 0 & 0 & 1 & 5 \\
\hline Urine CIE & 3 & 1 & 0 & (3) & 0 & 1 & 2 \\
\hline Sputum Gram stain & 5 & 0 & 0 & 0 & $(5)$ & 5 & 5 \\
\hline Sputum culture & 13 & 0 & 1 & 1 & 5 & (13) & 13 \\
\hline Sputum antigen & 26 & 6 & 5 & 2 & 5 & 13 & $(26)$ \\
\hline
\end{tabular}

CIE-counter immunoelectrophoresis.

morphology of the inclusion bodies under immunofluorescence was, however, typical of the TWAR strain. The patient from whom this was isolated died on the 11th day of his respiratory illness from acute myocardial infarction and convalescent serum was not available. No positive respiratory syncytial virus cultures were obtained despite there being an extensive concurrent epidemic among children.

\section{SEROLOGY}

Convalescent serum was obtained (three to four weeks after the acute serum specimen) in 80 of the surviving 86 patients $(93 \%)$. There were 17 serologically documented cases of $M$ pneumoniae, all with a fourfold antibody rise in both complement fixation and gel agglutination tests. There were many children with $M$ pneumoniae infection in the hospital during this epidemic.

Four patients had a diagnostic rise in antibody against Legionella species, but in none was an organism grown or antigen detected in sputum or nasopharyngeal aspirate specimens. Two had pneumococcal antigen in sputum and are classified as having a dual infection.

One patient with a prolonged hospital course, who on admission had $A$ calcoaceticus cultured from blood and influenza $B$ cultured from nasopharyngeal aspirate, had a fourfold rise in antibodies to Chlamydia trachomatis (serogroup ED).

Viral serology was diagnostic in four patients with a positive viral culture and added six further patients with diagnostic rises in antibody to influenza $A$ virus $\left(\mathrm{H}_{3} \mathrm{~N}_{2}\right)$ during the winter epidemic. Three of the latter patients probably had dual infections as they had sputum cultures of $S$ aureus, K pneumoniae and $S$ pneumoniae (one each).

On clinical grounds five patients may have had aspiration pneumonia. Two patients with epilepsy presented three and five days after a convulsion. No microbiological diagnosis was obtained in either patient. Three further patients, elderly men, with Gram negative bacilli cultured from their sputum may have aspirated; one had severe Parkinson's disease with bulbar disease, one had severe symptomatic oesophagitis, and the third had clinical and radiological evidence of aspiration on a barium swallow. Of the four patients with bronchiectasis and obvious new consolidation, two had probable $H$ influenzae infections and two no microbiological diagnosis.

Of the 27 patients with no microbiological diagnoses, 10 had received antibiotics $(37 \%)$ compared with 37 of the $65(57 \%)$ in whom a diagnosis was made. This, however, includes 17 patients with $M$ pneumoniae, 15 of whom had had antibiotics before admission, and among whom there were no dual diagnoses. Microbiological tests, particularly the tests for $S$ pneumoniae, were incomplete in the 27 patients with no microbiological diagnosis. No sputum specimen was obtained from eight, the sputum specimen was not analysed for antigen in five, and no urinary antigen tests were done in nine. Many of these patients may have had pneumococcal infection, as 19 of the 30 patients with that diagnosis were found by sputum analysis.

Among the six who died no microbiological diagnosis was made in two; in one the diagnosis was made at necropsy ( $S$ aureus grown by culture of lung tissue), and the remaining three had definite diagnoses before death ( $K$ pneumoniae, $S$ aureus, Chlamydia sp). There were no deaths due to $S$ pneumoniae.

\section{Discussion}

This study, though based on that of the British Thoracic Society study, ${ }^{1}$ differs in several respects. We wished to assess all cases of community acquired pneumonia admitted to our adult medical wards, so no age limits were set. Patients over 74 years of age made up one third of our survey group. The main difficulty experienced with respect to entry criteria for the very elderly was in determining whether pneumonia was the main reason for admission. Elderly patients provided all the cases of Gram negative bacillary pneumonia other than those with $H$ influenzae. Furthermore, eight of those over 74 resided in rest homes (these patients do not require 24 hour nursing, but are not fully independent). Two of the eight had Gram negative bacteriological diagnoses. The predilection for Gram negative bacillary pneumonia is well documented in elderly people ${ }^{23}$ and is related to oropharyngeal colonisation, particularly in association with diabetes and alcoholism ${ }^{4}$ and in those requiring institutional care. ${ }^{5}$

Despite inclusion of the very elderly, our modified prognostic index was found to be very useful, and is the outstanding feature of the study. Our modification allowed identification 
of high risk patients at admission. This high risk group may require a different treatment strategy or more invasive investigation, such as percutaneous lung aspiration or fibreoptic bronchoscopy, if mortality is to be improved. ${ }^{6}$

The early identification of the atypical organisms is difficult, with the possible exception of $L$ pneumophila. ${ }^{7}$ The $M$ pneumoniae specific IgM test may give a positive result in up to $86 \%$ of cases at the time of admission to hospital, ${ }^{8}$ but it remains negative in up to $15 \%$ of cases. ${ }^{1}$ We found that the gel agglutination test, though not diagnostic at admission, was diagnostic by about the 10 th day of the illness.

The yield of microbiological diagnoses $(72 \%)$ in our study is similar to that in other published series, ${ }^{19-12}$ though less than that of a Nottingham series. ${ }^{13}$ The results are very similar to those of the British Thoracic Society study $^{1}$; this is in part at least because the diagnostic criteria used are identical, because by coincidence our study also included an outbreak of $M$ pneumoniae, and because the use of antibiotics before hospital admission was very similar ( $51 \%$ here and $45 \%$ in the UK). Pneumococcal infection predominates in all studies, influenza $A$ is always in the top three or four causes, and the incidence of $M$ pneumoniae fluctuates regularly in three to five year cyclical outbreaks. The high incidence of legionella pneumonia in Nottingham of $15 \%^{8}$ has apparently not persisted and constitutes $2-5 \%$ of cases of community acquired pneumonia. $H$ influenzae is particularly associated with chronic lung disease, especially bronchiectasis, and whereas the British Thoracic Society study found no difference in incidence in chronic airflow obstruction, other studies suggest an increased incidence. ${ }^{14}$ Staphylococcal pneumonia is particularly associated with influenza epidemics. ${ }^{15}$

Among other causes, Chlamydia pneumoniae is currently receiving considerable attention. This newly described human pathogen, responsible for $6-10 \%$ of cases of pneumonia admitted to hospital, may also cause nosocomial pneumonia, and it occurs sporadically in epidemics. ${ }^{16}$ It is important because it does not respond to standard antibiotics. It causes a disease similar to $M$ pneumoniae - with upper and lower respiratory tract disease-and requires treatment with erythromycin or a tetracycline antibiotic, sometimes for a prolonged period of 10-14 days.

Viral respiratory tract infections, with the exception of influenza, are less common in adults than children, where respiratory syncytial virus regularly causes winter epidemics. Adenovirus is common among military conscripts, however, causing $22 \%$ of cases of community acquired pneumonia in one study. ${ }^{11}$

The use of pneumococcal antigen tests in recent studies of pneumonia has increased the yield of identified pneumococcal infection, but caution is necessary in the interpretation of sputum culture and antigen tests. Sputum is frequently "contaminated" by oropharyngeal organisms and various procedures have been advocated so that sputum findings may more closely resemble findings in transtracheal aspirates. ${ }^{1819}$ Furthermore, a study of 25 Navajo Indians found that, although sputum reflected the organisms isolated from lung aspirates as frequently as did transtracheal aspirates, multiple potential pathogens were recovered from all but five sputum specimens and from $27 \%$ of transtracheal aspirates. ${ }^{20}$ Consequently, where sputum culture and antigen tests were used $8-31 \%$ of patients with community acquired pneumonia have been reported as having dual infections. ${ }^{19-11}$ In a large study of 175 adults lung aspiration was performed in 144, a positive culture yielding in 90 of the cases and a change of treatment in 22 . Seventy four per cent of patients with positive blood or lung aspirate culture, or both, had sputum culture results that were either negative $(34 \%)$ or misleading in that another organism was isolated in $40 \%{ }^{21}$ The role of lung aspiration or bronchoscopy in pneumonia in patients at high risk of death has not been determined, and a similar outcome may be achieved by using a broad based treatment strategy for these patients rather than more invasive investigations.

Finally, a proportion of the mortality and morbidity of community acquired pneumonia may be avoided altogether by more widespread judicious use of influenza and pneumococcal vaccines. Of the 38 patients with definite or probable pneumococcal or influenzal infection in this study, 25 would have been eligible for vaccination against influenzal and pneumococcal disease. ${ }^{22}$ If both vaccines were $70 \%$ efficacious in reducing hospital admissions for these diseases, and if all 25 patients had received the vaccines, 17 admissions would have been prevented, a $19 \%$ reduction in admissions with pneumonia in this study. In previous surveys two thirds or more of patients with serious pneumococcal disease had been in hospital at least once three to five years before contracting pneumonia. ${ }^{22}$ of our 30 patients with pneumococcal infection 17 had had an admission to hospital within the last five years and almost all of those had a chronic illness or were over the age of 65 years.

We are grateful to Parke-Davis for funding the chlamydia studies, to Wellcome Diagnostics for providing pneumococcal antigen latex agglutination kits, and to the physicians of the department of medicine for allowing us to study their patients, and especially the medical registrars for enrolling patients. Special thanks are due to Ms K Walker for secretarial assistance.

1 Research Committee of the British Thoracic Society. Community acquired pneumonia in adults in British hospitals in 1982-1983: a survey of aetiology, mortality, prognostic factors and outcome. $Q J$ Med 1987;62:195-220.

2 Verghese A, Berk SL. Bacterial pneumonia in the elderly. Medicine (Baltimore) 1983;62:271-85.

3 Ebright JR, Rytel MW. Bacterial pneumonia in the elderly. J Am Geriatr Soc 1980;28:220-3.

4 Mackowiak PA, Martin RM, Jones SR, Smith JW. Pharyngeal colonization by Gram-negative bacilli in aspirationprone persons. Arch Intern Med 1978;138:1224-7.

5 Valenti WM, Trudell RG, Bentley DW. Factors predisposing to oropharyngeal colonization with Gram-negative bacilli in the aged. $N$ Engl J Med 1978;298:1108-11.

6 Harrison BDW, Farr BM, Connolly CK, et al for the Council of the British Thoracic Society. The hospital
C. 
management of community-acquired pneumonia. JR Coll Physicians Lond 1987;21:267-9.

7 Winter JH, McCartney AC, Fallon RJ, et al. Rapid diagnosis of an outbreak of legionnaires' disease at Glasgow Royal Infirmary. Thorax 1987;42:596-9.

8 Ali NJ, Sillis M, Andrews BE, Jenkins PF, Harrison BDW. The clinical spectrum and diagnosis of Mycoplasma pneumoniae infection. $Q J$ Med 1986;58:241-51.

9 Holmberg H. Aetiology of community-acquired pneumonia in hospital treated patients. Scand J Infect Dis 1987;19: 491-501.

10 Berntsson E, Blomberg J, Lagergard T, Trollfors B. Etiology of community-acquired pneumonia in patients requiring hospitalisation. Eur J Clin Microbiol Infect Dis requiring hospicali

11 Lehtomaki K, Leinonen M, Takala A, Hovi T, Herva E, Koskela M. Etiological diagnosis of pneumonia in military conscripts by combined use of bacterial culture and serological methods. Eur J Clin Microbiol Infect Dis 1988;7:348-54.

12 Ausina V, Coll P, Sambeat M, et al. Prospective study on the etiology of community-acquired pneumonia in children and adults in Spain. Eur J Clin Microbiol Infect Dis 1988;7:343-7.

13 Macfarlane JT, Finch RG, Ward MJ, Macrae AD. Hospital study of adult community-acquired pneumonia. Lancet 1982;ii:255-8.

14 Barnes DJ, Naraqi S, Igo JD. Haemophilus influenzae pneumonia in Melanesian adults: report of 15 cases.
Thorax 1987;42:889-91.

15 Woodhead MA, Radvan J, Macfarlane JT. Adult community-acquired staphylococcal pneumonia in the antibiotic era: a review of 61 cases. $Q J$ Med 1987;64:783-90.

16 Grayston JT, Wang SP, Kuo CC, Campbell LA. Current knowledge on Chlamydia pneumoniae, strain TWAR: an important cause of pneumonia and other acute respiratory diseases. Eur J Clin Microbiol Infect Dis 1989;8:191-202.

17 Cruz JR, Quinonez E, Fernandez A, Peratta F. Isolation of viruses from nasopharyngeal secretions: comparison of aspiration and swabbing as means of sample collection. $J$ Infect Dis 1987;156:415-6.

18 Geckler RW, Gremillion DH, McAllister CK, Ellenbogen C. Microscopic and bacteriologic comparison of paired sputa and trans-tracheal aspirates. J Clin Microbiol 1977; s:396-9.

19 Bartlett JG, Finegold SM. Bacteriology of expectorated sputum with quantitative culture and wash technique compared to transtracheal aspirates. Am Rev Respir Dis 1978;117:1019-27.

20 Davidson $\mathrm{M}$, Tempest B, Palmer DL. Comparison of sputum, transtracheal aspirates and lung aspirates. JAMA 1976;235:158-63.

21 Barnes DJ, Naraqi S, Igo JD. The role of percutaneous lung aspiration in the bacteriologic diagnosis of pneumonia in adults. Aust NZ J Med 1988;18:754-7.

22 Williams W, Hickson MA, Kane MA, et al. Immunization policies and vaccine coverage among adults. Ann Intern Med 1988;108:616-25. 